\title{
$\mathrm{CiSj}$
}

\section{TESTING OF MICROPROCESSOR DEVICES ON THE BASIS OF ARTIFICIAL NEURAL NETWORKS WITH CHANGEABLE PARAMETERS}

\author{
Viktor Lokazyuk ${ }^{1)}$, Viktor Cheshun ${ }^{2)}$, Vitaliy Chornenkiy ${ }^{3)}$
}

1) doctor, professor, Lvivska str. 11, Ternopil, 46000, Ternopil academy of a national economy, kism@beta.tup.km.ua 2) doctor, docent, Institutska str. 11, Khmelnitskiy, 29016, Technological university of Podillya, kism@beta.tup.km.ua

3) Institutska str. 11, Khmelnitskiy, 29016, Technological university of Podillya, kism@beta.tup.km.ua

\begin{abstract}
The base principles of a technique of application of 3-layer feedforward fullconnected artificial neural network for execution of adaptive algorithms of testing of digital microprocessor devices are considered. The method of change of weight coefficients and thresholds of artificial neurons in the mode of operation of artificial neural network realized at the hardware level is considered. The application of this method provides implementation of adaptive algorithms of testing of the large complexity with the limited hardware resources of artificial neural network.
\end{abstract}

Keywords: diagnosing, digital devices, adaptive algorithms of diagnosing, artificial neural network

\section{INTRODUCTION}

Increase of a degree of integration and the complication of an inner structure of digital microprocessor devices predetermines fixed rise of the requirements to their diagnosis equipment. It does the actual task of refinement existing and creation of new diagnosis equipment $[1,2]$.

Today by idiosyncrasy of the determined test diagnosing $[3,4]$ of digital microprocessor devices (DD) is wide application of adaptive (conditional) algorithms of testing. These algorithms provide purposeful definition of test vectors for output on the unit under test on the basis of the analysis previously of obtained returns. During testing it permits to reduce availability test sequences, used for installation of technical state of object, to diminish a common duration of testing and, as a consequence, to boost productivity of used technical equipment.

The essential rise of operating frequencies has stipulated increase of quantity of dynamic faults in modern DD [2,4]. Effective localization of faults of this type possible only at testing of object on maximum operating frequencies. The program processing of diagnostic data does not permit to reach necessary indicators of speed, therefore check of returns and choice of a way of continuation of the process of testing at execution of adaptive algorithms is assigned to hardware.

The existing equipment of complete hardware implementation of adaptive algorithms select a way of continuation of the process of testing as a result of comparing on coincidence of two values of vectors of returns. The first value is defined beforehand and is standard, and second value is obtained from unit under test. After each check by results of comparing is doing the choice of one of two possible ways of continuation of the process of testing. The model of such algorithm of diagnosing is the binary tree (fig. 1).

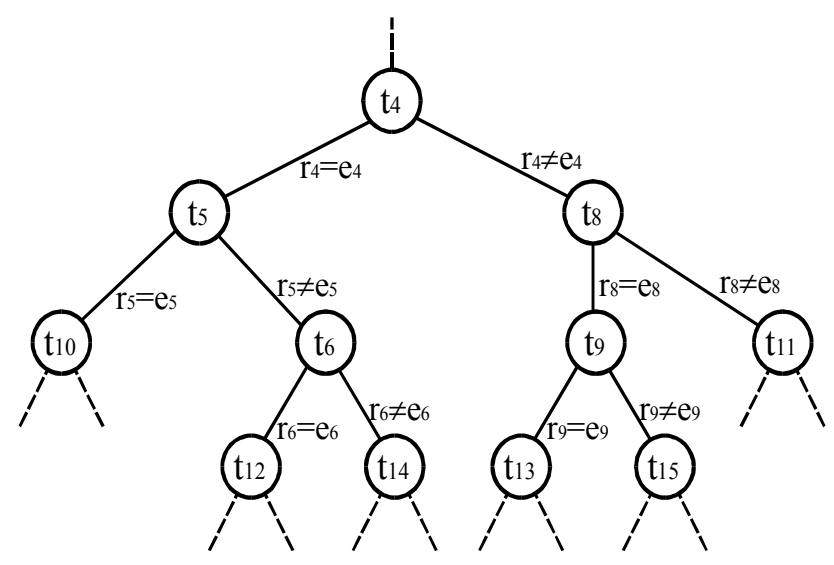

Fig. 1 - Fragment binary tree of adaptive algorithm.

The tops of a tree $\mathbf{t}_{\mathbf{i}}$ (Fig. 1) correspond to test vectors, which give on unit under test, and the arcs reflect possible transitions during testing depending on coincidence of obtained value of a vector of returns $r_{i}$ with standard $\mathbf{e}_{\mathbf{i}}$.

The shortcoming of such comparing is that it does not permit to take into account tags of manifestation of a fault, which have brought to an 
uncoincidence of values of vectors of returns.

The researches is displaying, that during testing at each stage the defined set of values of vectors of returns $\mathbf{R}_{\mathbf{i}}$ can receive, that do not coincide with standard value $\mathbf{e}_{\mathbf{i}}$. Thus, as a rule, $\left|\mathbf{R}_{\mathbf{i}}\right|>1$. Each values $\mathbf{r}_{\mathbf{i}, \mathbf{j}} \in \mathbf{R}_{\mathbf{i}}\left(1 \leq \mathbf{j} \leq\left|\mathbf{R}_{\mathbf{i}}\right|\right)$ characterizes possibility of existence unit under test in the defined technical states. Purposeful choice of a way of continuation of the process of testing for check of a hypothesis about finding of object in these states permits maximum to reduce necessary quantity of the tests. That is, the consideration of features of values of vectors of returns $\mathbf{r}_{\mathbf{i}, \mathbf{j}} \in \mathbf{R}_{\mathbf{i}}$ permits to select such way of continuation of the process of testing, which is by most effective in an appropriate case.

Let's designate a calculation (standard) set of vectors of returns, which can be obtained as a result of feed the test of a vector $\mathbf{t}_{\mathbf{i}}$ on unit under test, as $\mathbf{E}_{\mathbf{i}}$. Then the check of conditions of adaptive algorithm of testing will consist in matching on coincidence of obtained value of a vector of returns $\mathbf{r}_{\mathbf{i}}$ with set of standard values.

The fragment of a tree, which illustrates such adaptive algorithm of diagnosing, is given in a fig. 2 .

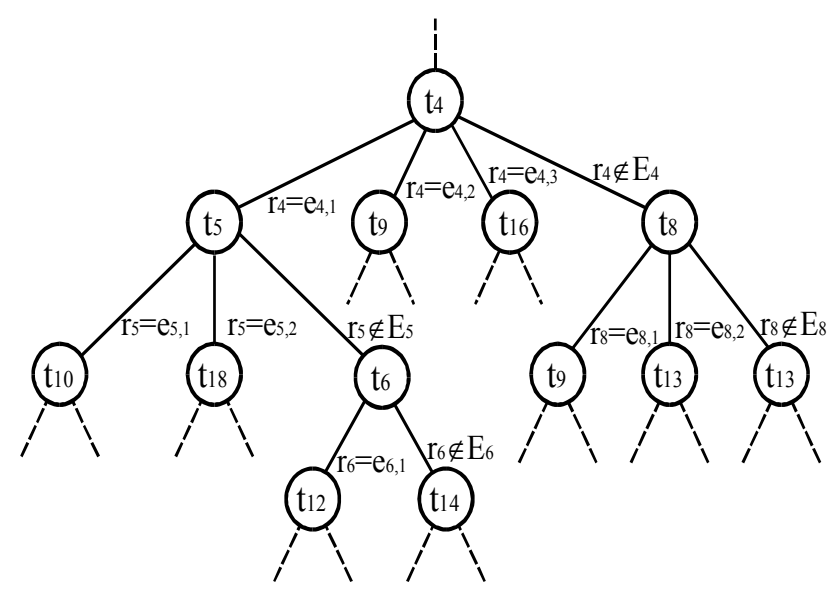

Fig. 2 - Fragment tree of adaptive algorithm.

Such approach permits by a maximum image to use advantages of adaptive algorithms of diagnosing to abbreviation of duration of the process of testing.

By problem, which arises at execution of adaptive algorithms of diagnosing according to the described approach, there is an implementation of multiple comparing of returnes.

Sequential comparing of the obtained value $\mathbf{r}_{\mathbf{i}}$ with units of set of standard values $\mathbf{E}_{\mathbf{i}}$, as well as program implementation of this process, do not provide of necessary metrics of speeds of technical diagnosis equipment.

The hardware implementation of parallel comparing of a vector of returnes $\mathbf{r}_{\mathbf{i}}$ of unit under test with standard values $\mathbf{e}_{\mathbf{i}, \mathbf{j}} \in \mathbf{E}_{\mathbf{i}}$ coupled with a complexity of prediction of necessary resources. The traditional approach with parallel comparing of standard values of vectors of returnes with separate blocks, obtained from the unit under test, is little effective. The quantity of standard values of returnes $\left(\left|\mathbf{E}_{\mathbf{i}}\right|\right)$ for different test vectors of a test sequence differs. This quantity also can vary during accumulation of data about the unit under test, differs for different types of unit under test and algorithms of their testing. It does not permit beforehand to forecast quantity of standard values, which can compare with a vector of returnes of unit under test by diagnosis equipment. Therefore in such situation it is heavy to define optimal quantity of the necessary blocks of comparing.

It has stipulated a urgency of search of the alternate approach to implementation of adaptive algorithms of testing by hardware. The researches, carried out by the writers, have shown, that by the effective tool for solution of this task are artificial neural networks.

\section{GENERAL DESCRIPTION OF A TECHNIQUE OF APPLICATION OF ARTIFICIAL NEURAL NETWORKS AT ADAPTIVE TESTING}

For implementation of adaptive algorithms of testing in view of features of returnes of the unit under test it is offered [5] to use 3-layer feedforward fullconnected artificial neural network (ANN). Hardware realized ANN (fig. 3) is a basis of the block of neural network control of process of testing.

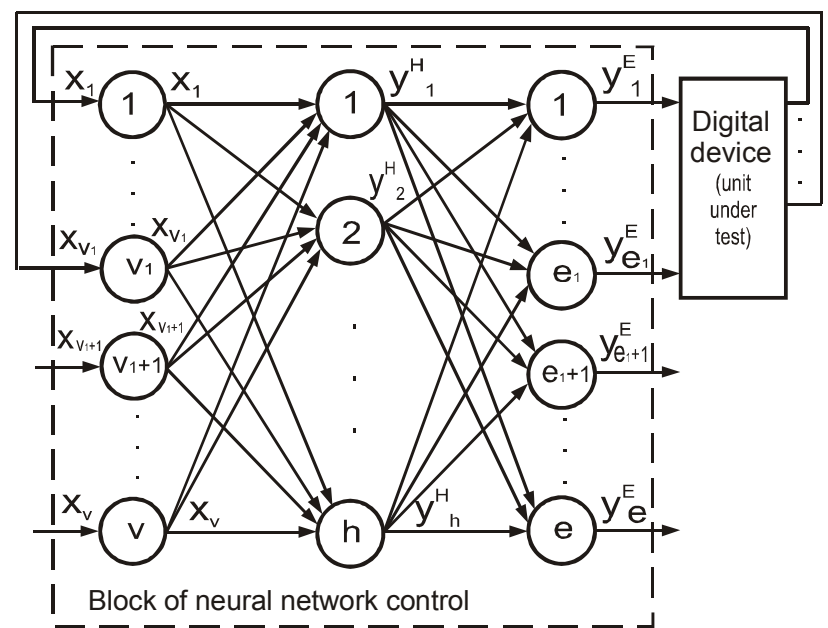

Fig. 3-Structural model $\overline{A N} \bar{N}$ with a scheme of connecting of the unit under test.

Input (receptor) layer of artificial neural network is intended for allocation of input signals on neurons of the hidden layer and not spend calculations.

The functions of processing of an input information and creation of output signals of the network are assigned to units of hidden and output (effector) layers, which in quality of the activate function use the threshold function: 


$$
f(x)=\left\{\begin{array}{l}
1, \text { if } x \geq 0 \\
0, \text { if } x<0
\end{array} .\right.
$$

Usage of the threshold function predetermines a homogeneity ANN and provides acceptance with its outputs of binary values of signals, traditional for digital circuitry.

The functions of output signals $\mathbf{y}$ of artificial neurons of hidden and effector layers are reflected by the formula:

$$
y=f\left(\sum_{i=1}^{n} x_{i} \cdot w_{i}-\theta\right) .
$$

where $\mathbf{x}_{\mathbf{i}}-\mathrm{i}$-th input signal of an artificial neuron, $\mathbf{i}=0,1, \ldots, \mathrm{n} ; \mathbf{x}_{\mathbf{i}} \in\{0,1\} ; \mathbf{w}_{\mathbf{i}}$ - weight coefficient of $\mathrm{i}$-th input of an artificial neuron; $\theta$ - threshold of an artificial neuron.

According to the offered technique ANN is realized hardware in structure of used diagnosing equipment.

Realization of training ANN at the hardware level is connected with substantial growth of cost of diagnosis resources. The support of such possibility is not essential necessity for execution of adaptive algorithms of testing. Therefore calculation of weight coefficients and thresholds of artificial neurons is fulfilled by program of simulation of operation ANN. Then values is calculated by program is loading in realized hardware ANN.

The technique of training ANN is founded on usage of a delta-rule [6].

Training ANN is fulfilled on possible values of returnes of unit under test. As a matter of fact, on each stage of training is used the appropriate set $\mathbf{E}_{\mathbf{i}}$. The elements of set $\mathbf{E}_{\mathbf{i}}$ for each test vector $\mathbf{t}_{\mathbf{i}}$ are fixed in view of features of manifestation of possible faults in returnes of unit under test.

The parameters of ANN are defined so that at arrival of any value $\mathbf{e}_{\mathbf{i}, \mathbf{j}} \in \mathbf{E}_{\mathbf{i}}$ on inputs of the network (signals $\left.\mathbf{X}_{1}-\mathbf{X}_{\mathbf{V} \mathbf{1}}\right)$ on its outputs $\left(\mathbf{Y}^{\mathbf{E}}{ }_{1}-\mathbf{Y}^{\mathbf{E}}{ }_{\text {e1 }}\right)$ the value of a test vector was shaped according to the requirements of adaptive algorithm of testing. Thus the input signals $\mathbf{X}_{\mathbf{V} \mathbf{1}+\mathbf{1}}-\mathbf{X}_{\mathbf{V}}$ are taken into account, which permit to distinguish identical returnes at different stages of diagnosing and fulfil a number of other functions. Also values of signals $\mathbf{y}^{\mathbf{E}}{ }_{\mathrm{e} 1+1}-\mathbf{y}^{\mathbf{E}}{ }_{\mathrm{e}}$ are defined necessary for operation of other blocks.

In further the loading of the calculated values of weight coefficients and thresholds of artificial neurons is fulfilled in realized hardware ANN. It provides its customization for execution of adaptive algorithm of testing.

The simulation of operation of the offered network during execution of adaptive algorithms of testing permits to detect an essential disadvantage, which can be considered common for all realized hardware artificial neural networks. It consists in limitation of functionalities from the point of view of training of the network through the rigidly defined structure of artificial neurons and links between them with limitation of a digit capacity of weight coefficients and thresholds. In case of complication of executable algorithms of testing it resultes to originating of situations, when from the defined moment the reaching of convergence becomes impossible at further training of ANN. It predetermines a problem of "overload" of ANN.

To prevent "overload" of ANN it is possible by a prior bookmark of redundancy, which can be considered as a reserve of resources for increase of functionalities of this network on a case of increase of complexity of the solving tasks (of adaptive algorithms of testing). It can be realized by three ways:

by increase of a significant digit capacity of weight coefficients of links and thresholds of artificial neurons. The disadvantage of this way is complication of realization of artificial neurons;

- by increase of quantity of layers of ANN. The disadvantage of this way is complication of a common inner structure of ANN;

by increase of quantity of neurons in internal layers of ANN. The disadvantage of this way is growth of internal layers of ANN and complication of realization of artificial neurons in other layers.

Any of the listed variants provides substantial growth of the hardware expenditures for realization of ANN, and accordingly, and increase of its cost price. Under such circumstances actual there is a task of definition of the optimal conciliatory proposal, as the insufficient reserve of resources of ANN does not permit to remove the indicated disadvantage in general, and redundant reserve generates a problem of substantial growth of the cost price of resources of diagnosing.

The feature of the process of implementation of adaptive algorithms of testing of modern DD is complexity of prediction of needs in resources for solution of the set tasks. The reason consists in prompt development of technologies of production of element base and digital engineering as a whole. The consequence is fixed rise of the requirements to possibilities of diagnosis equipment. Definition of optimal variant of reserve of resources of ANN under such circumstances is practically impossible. 


\section{TECHNIQUE AND EQUIPMENT OF CHANGE OF PARAMETERS OF ARTIFICIAL NEURAL NETWORK}

For elimination of a problem of "overload" of ANN the way is offered, according to which the increasing of its powers is fulfilled at the expense of giving of possibility of change of values of weight coefficients and thresholds of artificial neurons (further in the text - parameters of ANN) during operation. It provides the following differences in modes of training and operation of ANN and in structure of its units.

In common, the training of ANN is fulfilled by a delta-rule by a traditional way. In case the further training of neural network becomes impossible, the generated values of its parameters is stored as real for the given stage of execution of adaptive algorithm of testing. The training of the network for execution of the following part of algorithm continues after restoring initial values of its parameters.

Such approach provides decomposition of common algorithm of testing on several composites. For execution of any of them is used ANN with new properties (with used parameters). At decomposition of adaptive algorithm of testing on $\mathbf{m}$ of parts, as a matter of fact, is fulfilled $\mathbf{m}$ of procedures of training with calculate $\mathbf{m}$ of sets of parameters of ANN. Accordingly, the possibility of execution $\mathbf{m}$ of changes of parameters of ANN permits to decide the diagnostic tasks in $\mathbf{m}$ of time of the greater complexity. It can be considered as an alternate way of overgrowth of power of ANN.

Let's consider features of the hardware realization of change of parameters of ANN during operation. For giving of appropriate possibilities the artificial neurons of hidden and effector layers are supplemented by blocks of the registers for saving changed values of weight coefficients and thresholds. In a fig. 4 the indicated blocks consist from $\mathrm{m}$ of registers. It provides possibility to fulfil up to $\mathbf{m}$ of changes of parameters of ANN during implementation of adaptive algorithm of testing.

During preparation of ANN to execution of adaptive algorithm of testing the possible values of weight coefficients $\mathbf{w}_{\mathbf{i}}$ of all artificial neurons are written in the registers of saving of weight coefficients $\mathbf{R G}\left(\mathbf{w}_{\mathbf{i}}\right)$. Similarly in the registers of saving of thresholds $\mathbf{R G}(\Theta)$ of artificial neurons the possible values of thresholds $\Theta$ are written.

During preparation of ANN to execution of adaptive algorithm of testing the possible values of weight coefficients $\mathbf{w}_{\mathbf{i}}$ of all artificial neurons are written in the registers of saving of weight coefficients $\mathbf{R G}\left(\mathbf{w}_{\mathbf{i}}\right)$. Similarly in the registers of saving of thresholds $\mathbf{R G}(\Theta)$ of artificial neurons the possible values of thresholds $\Theta$ are written.

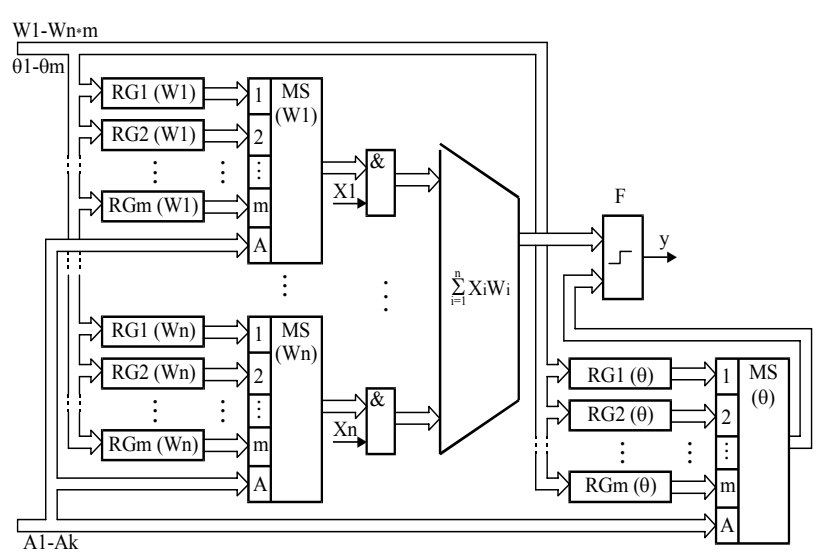

Fig. 4 - Structure of an artificial neuron.

During preparation of ANN to execution of adaptive algorithm of testing the possible values of weight coefficients $\mathbf{w}_{\mathbf{i}}$ of all artificial neurons are written in the registers of saving of weight coefficients $\mathbf{R G}\left(\mathbf{w}_{\mathbf{i}}\right)$. Similarly in the registers of saving of thresholds RG( $\Theta)$ of artificial neurons the possible values of thresholds $\Theta$ are written.

During operation of ANN parameters of an artificial neuron are defined by multiplexers of commutation of values of weight coefficients $\operatorname{MS}\left(\mathbf{w}_{\mathbf{j}}\right)$ and by multiplexer of commutation of values of thresholds $\mathbf{M S}(\Theta)$. These multiplexers select value from the registers $\mathbf{R G}\left(\mathbf{w}_{\mathbf{j}}\right)$ and $\mathbf{R G}(\Theta)$ under the control of signals of sampling $\mathbf{A}_{\mathbf{1}}-\mathbf{A}_{\mathbf{k}}$. On an artificial neuron the values of weight coefficients and thresholds is given which are necessary for execution of a preset stage of adaptive algorithm of testing.

The choice of the necessary values of weight coefficients and thresholds of artificial neurons requires the consideration of features of passing of the process of testing (according to the requirements to implementation of adaptive algorithm). Therefore functions of creation of signals of sampling $\mathbf{A}_{\mathbf{1}}-\mathbf{A}_{\mathbf{k}}$ rely on ANN.

The possibility of control of change of parameters of ANN is reached by rather small increase of its hardware complexity (in matching with other considered ways of increasing of functionalities). For this purpose it is enough to supplement a effector layer by artificial neurons in quantity equal to number of a management signals by multiplexers $\left(\log _{2} \mathbf{m} \leq \mathbf{k}<\mathbf{1}+\log _{2} \mathbf{m}\right)$. In reflected in a fig. 3 of model for control of multiplexers the part of signals $\mathbf{y}^{\mathbf{E} 1+1^{-}}-\mathbf{y}^{\mathbf{E}}$ can be used.

The positive feature of such modernization of ANN is also absence of need of development of specialized artificial neurons or modifications existing. For support of execution of control functions by change of parameters of ANN it the effector layer is supplemented by typical artificial 
neurons. Value of weight coefficients and thresholds of these neurons are defined on a stage of training so that they during operation of ANN shaped necessary values of signals of control of multiplexers $\mathbf{A}_{\mathbf{1}}-\mathbf{A}_{\mathbf{k}}$. If during the defined period at execution of adaptive algorithm of testing of necessity in change of parameters of the network is not present, value on outputs of these artificial neurons of a effector layer do not vary.

\section{CONCLUSION}

The offered technique of application of artificial neural networks permits to boost efficiency of realization of adaptive algorithms of testing of modern digital devices. The given technique is founded on usage of ability of artificial neural networks simultaneously to check several conditions of adaptive algorithm of testing. It permits to analyse returnes of the unit under test in view of features of manifestation of possible faults and to select the best way of continuation of the process of testing. In result the quantity of the tests necessary for definition of a state of the unit under test diminishes and the duration of the process of testing is reduced. The hardware realization of artificial neural networks permits to reach high indicators of speed of diagnosis equipment that is important for search of dynamic faults.

Change of weight coefficients and thresholds of artificial neurons eliminate the disadvantage of hardware artificial neural networks, which consists in limitation of functionalities by a rigid inner structure. For implementation of change of parameters of artificial neurons in the mode of operation of artificial neural networks is developed hardware. They permit at the expense concerning small increase of internal complexity of artificial neural networks to fulfil adaptive algorithms of testing in several times more complex, than without change of parameters.

The technique of change of weight coefficients and thresholds of artificial neurons in the mode of operation of artificial neural networks can be effectively used for increase of functionalities of hardware artificial neural networks at solution of other tasks.

\section{REFERENCS}

[1] V. Lokazuk.

Microprocessors

and microcomputer in productions. TUP, Khmelnitskiy, 2000. p. 226.

[2] V. Lokazuk, O. Pomorova, A. Dominov. Intellectual diagnosing of microprocessor devices and systems. Taki spravy, Khmelnitskiy-Kyiv, 2001. p. 286.
[3] V. Lokazuk,
V. Karyakin.
Combined

diagnosing and reliability of computing devices. Podillya, Khmelnitskiy, 1994. p. 156.

[4] V. Lokazuk. Control and diagnosing of computing devices and systems. TUP, Khmelnitskiy, 1996. p. 175.

[5] V. Chornenkiy, V. Cheshun. Implementation of polihotonomical procedures of diagnosing of digital devices by artificial neural networks, The Bulletin of Technological University of Podillya (1) (2001). p. 202-206.

[6] F. Uosermen. Neural computing technique. Mir, Moscow, 1992. p. 233.

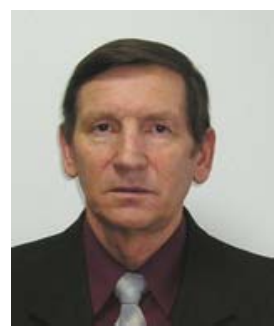

Viktor Lokazuk - Doctor, Professor, Dean of Faculty of Computer Systems and Programming of Khmelnitskiy National university, academician of the International Academy of information and International Academy of computer sciences and systems. In 1995 has protected the doctor's dissertation on a subject: "Methods and means of the combined diagnosing of digital structures with components of the raised degree of integration". Area of scientific interests is development of methods and means of intellectual diagnosing of digital devices and systems.

Viktor Cheshun - doctor, Assistant Professor of Computer Systems Department of Khmelnitskiy National university. In 1999 has protected the candidate dissertation on a subject: "Optimization of sequences the test - vector during the testing combined

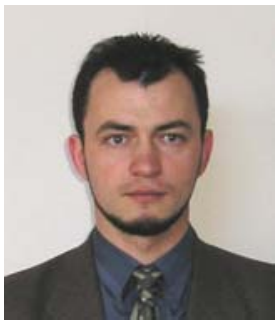
diagnosing of digital microprocessor devices". The area of scientific interests is research of prospects of use artificial neural networks for localization of malfunctions of a dynamic type of digital devices and systems.

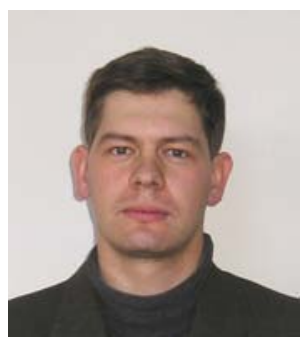

\section{Vitaliy Chornenkiy}

Doctor, Assistant Professor of Computer Systems Department of Khmelnitskiy National University. In 2003 has protected the candidate dissertation on a subject: "Resources of test diagnosing of digital devices on the basis of artificial neural networks". The area of scientific interests is development of means of diagnosing of digital devices on the basis of artificial neural networks. 